\title{
Ergosterol peroxide inhibits ovarian cancer cell growth through multiple pathways
}

\author{
This article was published in the following Dove Press journal: \\ OncoTargets and Therapy \\ 13 July 2017 \\ Number of times this article has been viewed
}

\author{
Weiwei $\operatorname{Tan}^{1, *}$ \\ Meihong Pan ${ }^{1, *}$ \\ Hui Liu' \\ Hequn Tian' \\ Qing Ye' \\ Hongda Liu ${ }^{2}$ \\ 'Department of Traditional Chinese \\ Medicine, Yidu Central Hospital of \\ Weifang, Weifang, Shandong, China; \\ ${ }^{2}$ Department of General Surgery, \\ Qilu Hospital Affiliated to Shandong \\ University, Jinan, Shandong, China \\ *These authors contributed equally \\ to this work
}

\begin{abstract}
Ergosterol peroxide (EP), a sterol derived from medicinal mushrooms, has been reported to exert antitumor activity in several tumor types. However, the role of EP toward ovarian cancer cells has not been investigated. In this study, we analyzed the cytotoxicity of EP in various cell lines representing high-grade serous ovarian cancer and low-grade serous ovarian cancer, respectively. Although EP showed no significant inhibition of the viability of normal ovarian surface epithelial cells, it impaired the proliferation and invasion capacities of tumor cells in a dose-dependent manner. We further figured out key modulators involved in its antitumor effects by quantitative reverse transcription polymerase chain reaction, ELISA, and Western blot. The nuclear $\beta$-catenin was down-regulated upon EP treatment, subsequently reducing the Cyclin D1 and c-Myc expression levels. Meanwhile, the protein level of protein tyrosine phosphatase SHP2 was up-regulated in EP treated cells, whereas Src kinase activity was inhibited. Both activation of SHP2 phosphatase and inhibition of Src kinase decreased the phosphorylation level of transducer and activator of STAT3 protein, which was implicated in oncogenesis. On the other hand, EP remarkably inhibited the expression and secretion of VEGF-C, implying its involvement in counteracting tumor angiogenesis. Moreover, EP treatment showed comparable cytotoxic effect with $\beta$-catenin knock-down or STAT3 inhibition. Taken together, our results demonstrated that EP showed antitumor effects toward ovarian cancer cells through both $\beta$-catenin and STAT3 signaling pathways, making it a promising candidate for drug development.
\end{abstract}

Keywords: $\beta$-catenin, ergosterol peroxide, ovarian cancer, STAT3

\section{Introduction}

Ovarian cancer is the most lethal gynecologic malignancy due to the advanced stage at the time of diagnosis for most patients. ${ }^{1} \mathrm{Up}$ to now, surgical resection plus postoperative chemotherapy has been the most popular treatment. ${ }^{2}$ Among various chemotherapies, platinum combined with paclitaxel is now the first-line therapy for ovarian cancer. ${ }^{3}$ However, a large number of patients may develop drug resistance, which is a major cause for the unsatisfactory disease prognosis. ${ }^{4}$ Therefore, increasing studies are focusing on elucidating chemo-resistance mechanisms as well as developing novel agents.

Natural products have been recognized to have great potential in treating various diseases including cancer, ${ }^{5}$ and studies searching for active natural products are drawing more and more attention. Medicinal mushroom is a prime example of discovering natural products, ${ }^{6}$ with Ganoderma lucidum the most well-known one. Certain components from G. lucidum have been reported to be beneficial for treating inflammation and cancer. ${ }^{7-10}$ Ergosterol peroxide (EP), a $\beta$-D-glucan metabolite, is an important component in $G$. lucidum as well as in other medicinal mushrooms. EP has been reported to possess potential antitumor activity toward various cancer types, including prostate cancer, ${ }^{11}$
Correspondence: Hongda Liu Department of General Surgery, Qilu Hospital Affiliated to Shandong University, 107 Wenhua Xi Road, Jinan,

Shandong 250012 , China

Tel +86 4l 24179005

Fax +8653182169114

Email liuhongda188@I63.com
OncoTargets and Therapy 2017:10 3467-3474

(c) (i) (5) 2017 Tan et al. This work is published and licensed by Dove Medical Press Limited. The full terms of this license are avaliable at https://www.dovepress.com/terms.php cc. hereby accept the Terms. Non-commercial uses of the work are permitted without any further permission from Dove Medical Press Limited, provided the work is properly attributed. For permission for commercial use of this work, please see paragraphs 4.2 and 5 of our Terms (https://www.dovepress.com/terms.php). 
colorectal cancer, ${ }^{12}$ hepatocellular cancer, ${ }^{13}$ and myeloma. ${ }^{14}$ However, the effects of EP toward ovarian cancer cells have not been investigated.

In the current study, we initially examined the effects of EP toward different cell lines representing both high-grade serous ovarian cancer (HGSOC) and low-grade serous ovarian cancer (LGSOC), respectively. Our results demonstrated that EP can significantly inhibit the proliferation and migration of ovarian cancer cells, while showing little effect on normal ovarian surface epithelial (OSE) cells. Further studies revealed that EP can induce at least two antitumor pathways in ovarian cancer cells, one is the $\beta$-catenin-c-Myc/ Cyclin D1 pathway and the other is SHP2/Src-STAT3-VEGF pathway. Our study provided evidence for the potency of EP in drug development targeting ovarian cancer.

\section{Materials and methods}

\section{Reagents}

Purified EP was purchased from ChemFaces (Wuhan, China). STAT3 inhibitor S3I-201 was purchased from Sigma-Aldrich Co. (St Louis, MO, USA). Presto Blue cell viability reagent and Lipofectamine ${ }^{\circledR} 2000$ reagent were obtained from Thermo Fisher Scientific (Waltham, MA, USA). All the antibodies for Western blot were purchased from Santa Cruz Biotechnology Inc. (Dallas, TX, USA): $\beta$-catenin (sc-65480), SHP2 (sc-280), pT419-Src (sc-81521), Src (sc-130124), pS727-STAT3 (sc-8001-R), STAT3 (sc-293151), $\beta$-actin (sc-58673). Primers for reverse transcription polymerase chain reaction (RT-PCR) were purchased from BGI (Beijing, China). Enzyme linked immunosorbent assay (ELISA) kit for VEGF-C was purchased from Thermo Fisher Scientific.

\section{Cell culture}

In this study, we collected OVCAR-3 and CAOV3 cells as study models representing HGSOC, and selected HOC-7 and MPSC-1 to represent LGSOC. In addition, we purchased OSE cells as control cells to verify whether EP has specific anticancer activity or whether it would kill healthy cells. All the cells were obtained from Tumor Cell Bank Research Institute of the Chinese Academy of Medical Sciences. The cells were cultured in RPMI-1640 containing 10\% (v/v) fetal bovine serum (FBS) with $10 \mu \mathrm{g} / \mathrm{mL}$ penicillin/streptomycin. All cells were incubated in a humidified atmosphere with $5 \% \mathrm{CO}_{2}$ at $37^{\circ} \mathrm{C}$ and used for studies in the logarithmic phase of growth.

\section{Cytotoxicity}

Presto Blue ${ }^{\circledR}$ assay was used to assess cytotoxicity of EP on cells. Cells were seeded in 96-well plates at a density of 5,000 cells/well and cultured overnight for adhesion.
The cells were then treated with different concentrations of $\mathrm{EP}(0,12 \mu \mathrm{M}, 25 \mu \mathrm{M}, 50 \mu \mathrm{M}$, and $100 \mu \mathrm{M})$ for $48 \mathrm{~h}$ to test the dose response.

For further cytotoxic analysis, $50 \mu \mathrm{M}$ EP was chosen as the final treatment concentration. After incubating with EP for a designated period, Presto Blue reagent was added and the mixture was incubated for $3 \mathrm{~h}$ followed by fluorescence measurement by excitation at $535 \mathrm{~nm}$ and emission at $595 \mathrm{~nm}$. One percent dimethyl sulphoxide (DMSO) was added into medium as negative control.

\section{Scratch closure migration assay}

The cell mobility was evaluated by scratch assays. ${ }^{15}$ Briefly, cells were grown to $80 \%-90 \%$ confluence in $10 \mathrm{~mm}^{2}$ tissue culture dishes, then medium was removed and a scratch was made at the middle of the dish with a $100 \mu \mathrm{L}$ pipette tip. Then, the dishes were slightly washed and EGF containing medium with $50 \mu \mathrm{M}$ EP or $1 \%$ DMSO was added. After $24 \mathrm{~h}$ incubation, cell migration was monitored under a microscope. The wound area covered by migrated cells was presented in percentage $(\%)$.

\section{Subcellular fractionation, protein collection, and Western blot}

Cells were treated with or without EP for $16 \mathrm{~h}$, then protein was collected. Briefly, cells were scratched and harvested by centrifugation at $1,000 \mathrm{rpm}$ for $2 \mathrm{~min}$ at $4^{\circ} \mathrm{C}$, then cells were lysed using ice-cold lysis buffer supplemented with protease inhibitors and phosphatase inhibitors. After centrifugation and disposal of the precipitate, proteins were denatured with $2 \%$ sodium dodecyl sulfate (SDS), electrophoretically resolved on $10 \%$ SDS-polyacrylamide gel electrophoresis gels, and transferred onto a nitrocellulose membrane. After blocking the non-specific binding sites with 5\% bovine serum albumin, the membrane was probed with diluted primary antibodies $(1: 1,000)$ at $4^{\circ} \mathrm{C}$ overnight. Membranes were then washed and further incubated with the peroxidaseconjugated secondary antibody (1:5,000 dilution) for $1 \mathrm{~h}$ at room temperature. The immune-bands were detected using the enhanced chemiluminescence reagents. The images were scanned and semi-quantified using Image J software.

To test nuclear $\beta$-catenin, subcellular fractionation was performed. Briefly, cells were collected and resuspended using hypotonic lysis buffer (10 mM HEPES/10 mM NaCl/1 mM $\mathrm{KH}_{2} \mathrm{PO}_{4} / 5 \mathrm{mM} \mathrm{NaHCO} / 1 \mathrm{mM} \mathrm{CaCl}$ ), then dounced to homogenize cells. Cell lysates were centrifuged at 1,500 rpm for $5 \mathrm{~min}$. The pellet from the centrifugation was collected and washed twice and resuspended in nuclear extraction buffer (10 mM Tris, pH 7.5/300 mM sucrose/0.1\% NP-40). 
The pellet was also dounced 20 times to homogenize nucleus and then spun down. The pellet was finally resuspended with nuclear isolation buffer containing $1 \%$ Triton X-100 to generate the nuclear fraction. Western blot was used to evaluate the protein level of nucleus $\beta$-catenin as described previously.

\section{VEGF-C quantification by ELISA}

The expression and release of VEGF-C in cell culture medium was quantified by a specific VEGF-C ELISA kit. After being stimulated with EGF and further treated in the presence or absence of EP for $16 \mathrm{~h}, 1 \mathrm{~mL}$ of medium was collected and filtered with a $10 \mathrm{kD}$ concentrator. The manufacturer's protocol for ELISA (Thermo Fisher Scientific) was followed. ${ }^{16}$ Samples were evaluated at OD $450 \mathrm{~nm}$ by a microplate reader. The fold changes of VEGF-C production between control group and EP-treated group were calculated.

\section{Quantitative reverse transcription polymerase chain reaction (RT-qPCR)}

After serum starvation for $24 \mathrm{~h}$, the cells were stimulated with EGF for $16 \mathrm{~h}$ with or without EP treatment. Total RNA was extracted using TRIzol reagent (Thermo Fisher Scientific) according to the manufacturer's instructions. The RNA levels of Cyclin D1 and c-Myc were then analyzed by RT-qPCR. Briefly, reverse transcription was performed with $1 \mu \mathrm{g}$ total RNA using high capacity cDNA-RT kit. Then the qPCRs were performed using TaqMan gene expression assay kit. ${ }^{17}$ The primers were as follows:

Cyclin D1: 5'-GTC TTC CCG CTG GCC ATG AAC TAC-3' and 5'-GGA AGC GTG TGA GGC GGT AGT AGG-3';

c-Myc: 5'-CAA GAG GCG AAC ACA CAA CGT C-3' and 5'-CTG TTC TCG TCG TTT CCG CAA C-3';

$\beta$-actin: 5'-TCA TCA CCA TTG GCA ATG AG-3' and 5'-CAC TGT GTT GGC GTA CAG GT-3'.

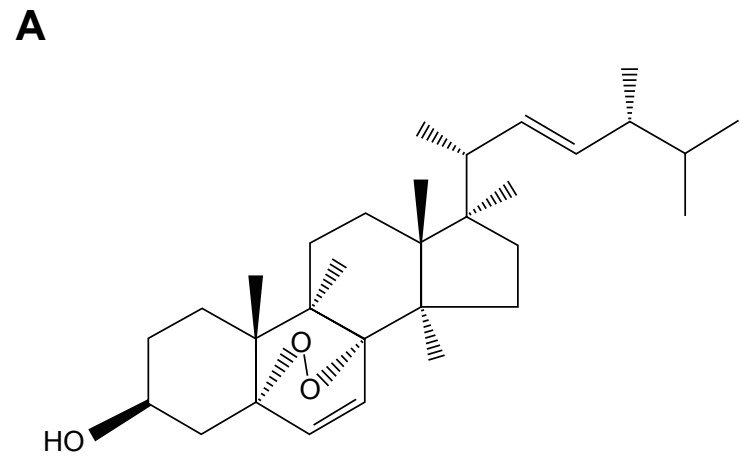

\section{Cell transfection}

Cells were transfected with plasmids or siRNAs to confirm the involvement of $\beta$-catenin and STAT3 in EP-induced antitumor effects. The $\beta$-catenin-pcDNA3 (catalog no 16828) and STAT3-pcDNA3 (catalog no 74433) plasmids were both purchased from Addgene. The $\beta$-catenin-siRNA was ordered from Sigma-Aldrich Co. with the following sequence: 5'-AGC UGA UAU UGA UGG ACA GdTdT-3'. All transfection experiments were carried out with Lipofectamine 2000 reagent following the manufacturer's instructions.

\section{Statistical analysis}

Each experiment was independently conducted at least three times. Bars in all figures indicate the mean and the SD. Student's $t$-test was performed to analyze statistical differences between the two groups; one-way ANOVA test was used to compare the differences among multiple groups. A $P$-value $<0.05$ was considered statistically significant.

\section{Results EP impairs proliferation and migration of ovarian cancer cells}

To investigate the cytotoxicity of EP (Figure 1A) on ovarian cancer cells, we firstly evaluated the dose-response of EP toward different cell lines (Figure 1B). Although EP could inhibit the cell proliferation of all tested cell lines in a dose-dependent manner, CAOV3 cells showed the highest sensitivity while the other three cell lines needed a higher EP concentration $(50 \mu \mathrm{M})$ to substantially inhibit cell viability. The different sensitivity to EP treatment reflects the prominent gene heterogeneity of ovarian cancer.

We then performed time-dependent cell viability assay after treating the cells with $50 \mu \mathrm{M}$ EP or $1 \%$ DMSO as control. Our results showed that EP had little effect on the

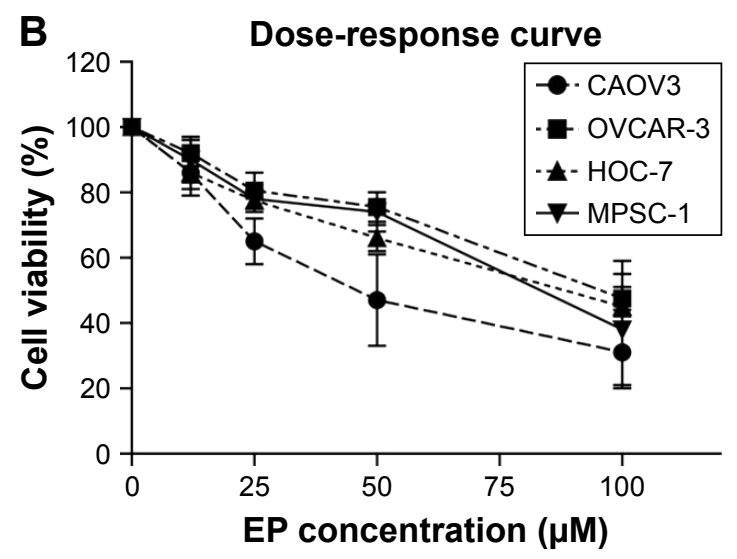

Figure I Chemical structure of ergosterol peroxide (EP) and dose-response curve.

Notes: (A) Chemical structure of EP. (B) Dose-response curve of EP toward different ovarian cancer cell lines after being treated for $48 \mathrm{~h}$. 

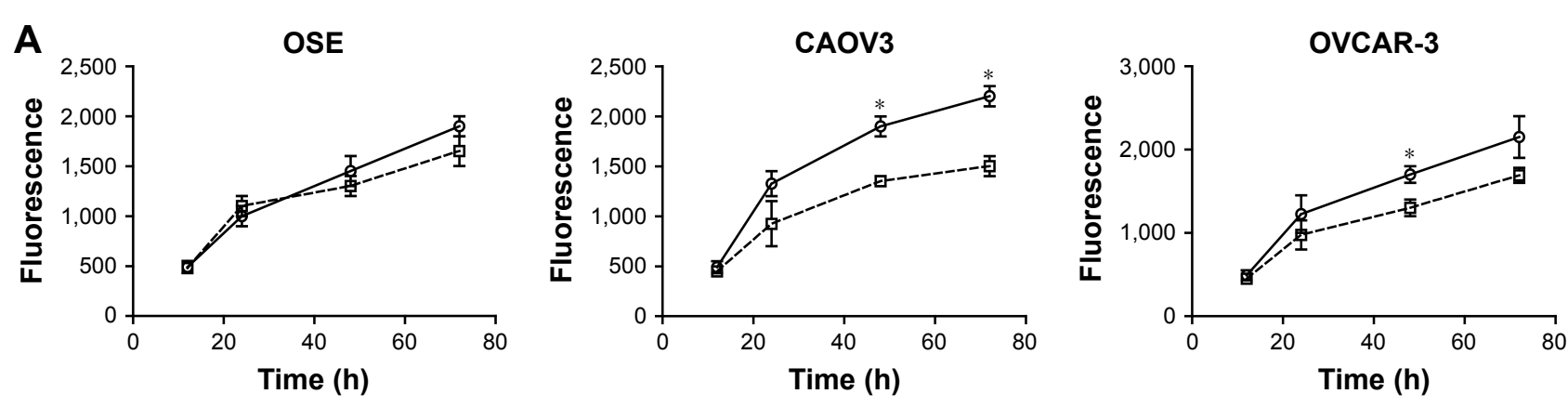

HOC-7
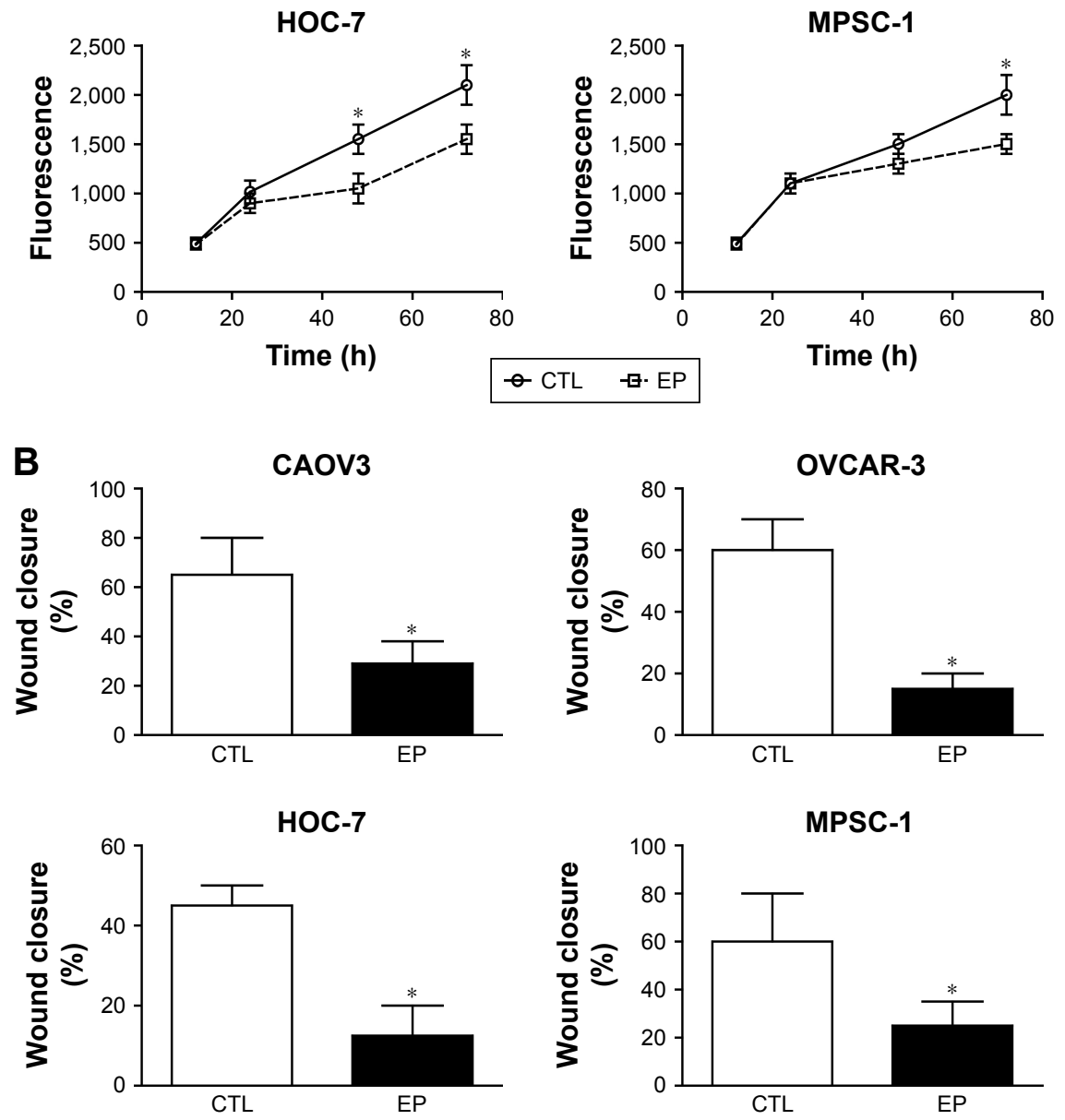

Figure 2 EP inhibits cell viability and mobility of ovarian cancer cells.

Notes: $(\mathbf{A})$ EP $(50 \mu \mathrm{M})$ showed little effect on the cell viability of OSE cells, but significantly inhibited the proliferation of ovarian cancer cell lines. (B) The migration ability of ovarian cancer cells was also down-regulated by EP treatment. Data were from three independent experiments and are presented as mean \pm SD. Student's $t$-test was performed to analyze the statistical differences between two groups. $* P<0.05$.

Abbreviations: OSE, ovarian surface epithelial; CTL, control; EP, ergosterol peroxide.

viability of normal OSE cells (Figure 2A). However, it significantly inhibited the proliferation of both HGSOC and LGSOC cell lines after $48 \mathrm{~h}$ treatment (Figure 2A).

In addition, we carried out a wound healing assay to evaluate the changes of cell migration capacity upon EP treatment. Consistent with cell viability results, the scratch experiments showed that EP impaired the migration of ovarian cancer cells (Figure 2B). The impaired proliferation and migration capacities with EP treatment revealed its remarkable potency in antitumor drug development.

\section{EP down-regulates the nuclear} translocation of $\beta$-catenin and expression of target proteins

Deregulation of $\beta$-catenin is one of the general mechanisms in cancer proliferation and aggression, ${ }^{18}$ therefore, we further 
investigated whether EP can modulate the $\beta$-catenin pathway in ovarian cancer cells. Western blot results demonstrated that EP treatment reduced the nuclear $\beta$-catenin protein level (Figure 3A) in both HGSOC and LGSOC cell lines.

Nuclear translocation of $\beta$-catenin can promote the transcription and expression of various oncogenic genes; here we chose Cyclin D1 and c-Myc as the biomarkers for further cellular assays. Consistently, the mRNA levels of Cyclin D1 (Figure 3B) and c-Myc (Figure 3C) were both downregulated in the four ovarian cancer cell lines.

\section{EP inhibits STAT3 signaling by regulating Src and SHP2}

STAT protein family, especially STAT3, is one of the most promising targets for cancer therapy including ovarian cancer. ${ }^{19,20}$ Moreover, it has been previously reported that EP can modulate the phosphorylation status of STAT3 protein in myeloma cells. ${ }^{14}$ In this study, we firstly tested the effects of EP on STAT3 pathway in ovarian cancer cells.

Our results showed that EP significantly down-regulated the phosphorylation level of STAT3 with a slight effect on
A

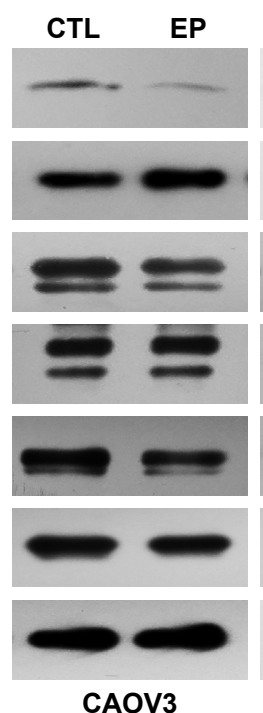

CAOV3

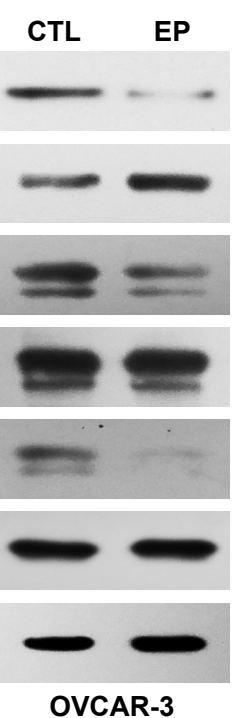

OVCAR-3

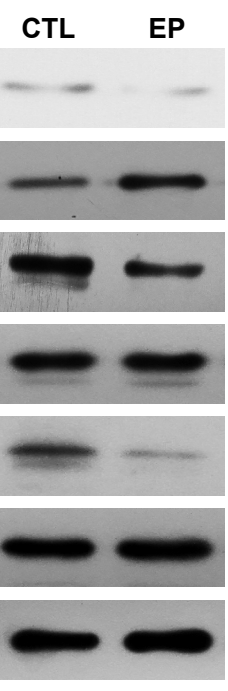

HOC-7
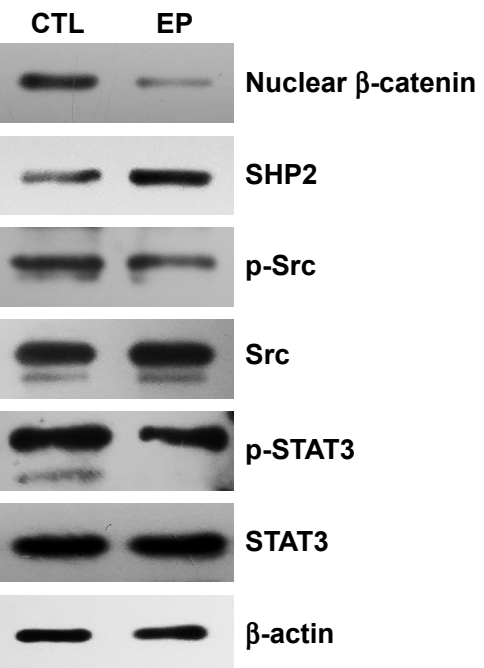

MPSC-1
B

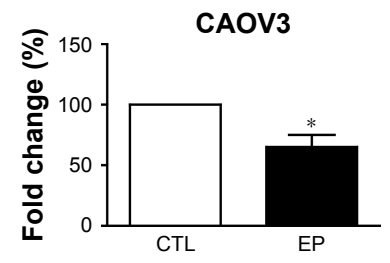

C

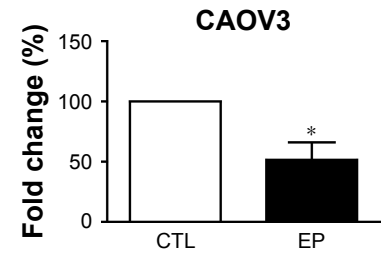

D

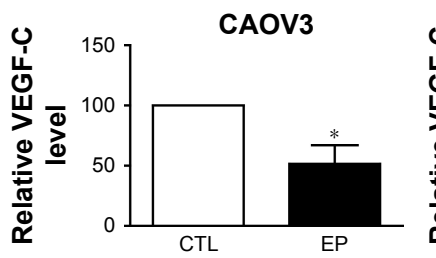

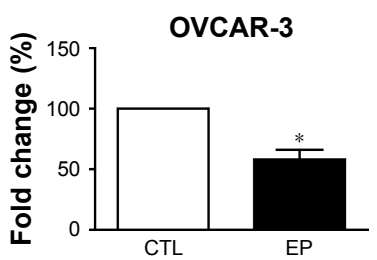

OVCAR-3

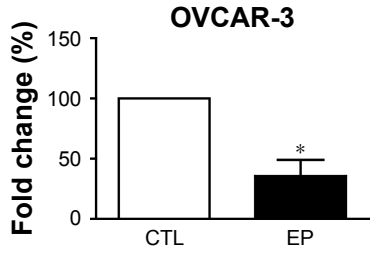

OVCAR-3

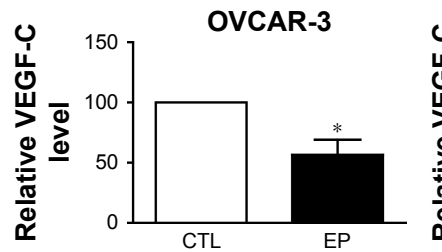

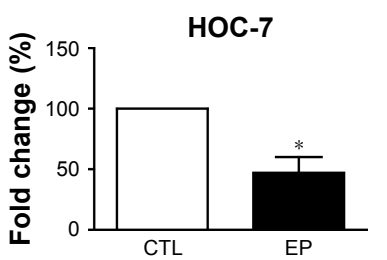

HOC-7

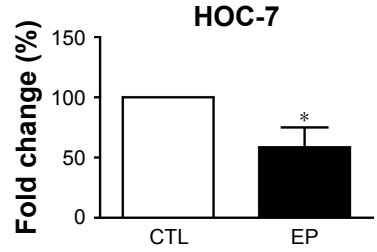

HOC-7

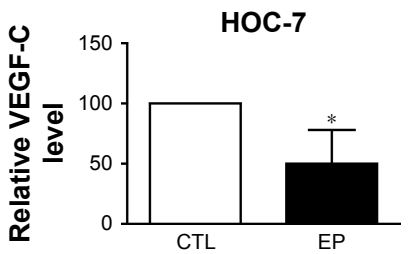

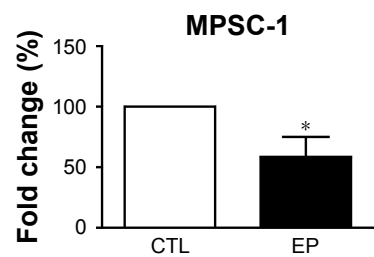

MPSC-1

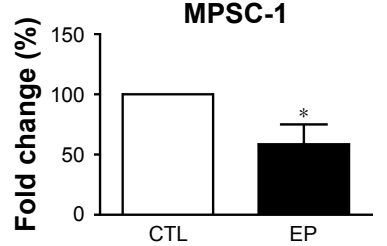

MPSC-1

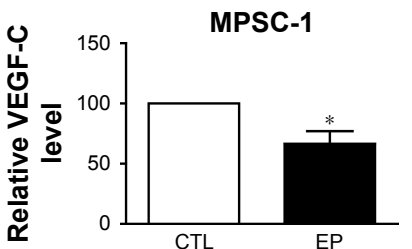

Figure 3 EP inhibits the oncogenic signaling mediated by $\beta$-catenin and STAT3.

Notes: (A) EP $(50 \mu \mathrm{M})$ treatment induced changes of oncogenic proteins on both expression level and post-translational modification level. The mRNA levels of Cyclin DI (B) and c-Myc (C) were decreased after EP treatment for $16 \mathrm{~h}$. ELISA results showed that the production of VEGF-C, an important protein in STAT3 pathway, was also inhibited upon EP treatment (D). $* P<0.05$.

Abbreviations: CTL, control; ELISA, enzyme linked immunosorbent assay; EP, ergosterol peroxide. 
total STAT3 protein level (Figure 3A). Src kinase and SHP2 phosphatase are two well-known up-stream modulators of STAT3, which can regulate its phosphorylation and activity. ${ }^{20,21}$ Therefore, we next tested the changes of these two proteins. Western blot results revealed that the SHP2 expression level was increased under EP treatment; meanwhile, the phosphorylation of Src was decreased, even the total Src protein level showed no significant change (Figure 3A). Furthermore, the production of VEGF-C, a wellrecognized effector of STAT3 pathway, was also inhibited by EP treatment (Figure 3D).

To further confirm the involvement of $\beta$-catenin and STAT3 in EP's effects. We performed the knock-in assay for $\beta$-catenin and STAT3; both showed enhanced cell viability compared with control cells (Figure 4A). On the other hand, knock-down of $\beta$-catenin by siRNA or inhibition of STAT3 by specific inhibitor (S3I-201) significantly inhibited cell proliferation. Moreover, treatment with EP showed comparable cytotoxic effect with $\beta$-catenin-siRNA or STAT3 inhibition (Figure 4B), suggesting that $\beta$-catenin and STAT3 are both implicated in the antitumor mechanisms (Figure 4C).

\section{Discussion}

G. lucidum is now regarded as an antitumor drug in traditional Chinese medicine. ${ }^{22}$ For example, G. lucidum extractions were reported to have hepatoprotective effects and induce apoptosis of HepG2 cells. ${ }^{23}$ EP is a natural product present in G. lucidum, which has been shown to regulate various biological processes including oxidation, ${ }^{12}$ inflammation, ${ }^{24}$ and tumor cell growth. ${ }^{25}$

In the past decade, the effects of EP extracted from G. lucidum have been studied in various tumor types. . $^{911,26,27}$ The results of the present study indicate that EP suppresses $\beta$-catenin-induced transcription of Cyclin D1 and c-Myc in ovarian cancer cells. Meanwhile, our study shows the effects of EP on regulating Src/SHP2-STAT3 pathway, indicating the multiple functions of EP in suppressing tumor growth. Consequently, the proliferation and migration of ovarian cancer cells were also inhibited upon EP treatment.

Intriguingly, we found that EP can attenuate the expression and secretion of VEGF-C, which has been well-recognized as a positive modulator of angiogenesis. ${ }^{28,29}$ In addition, it has been reported that constitutive STAT3 activation contributed
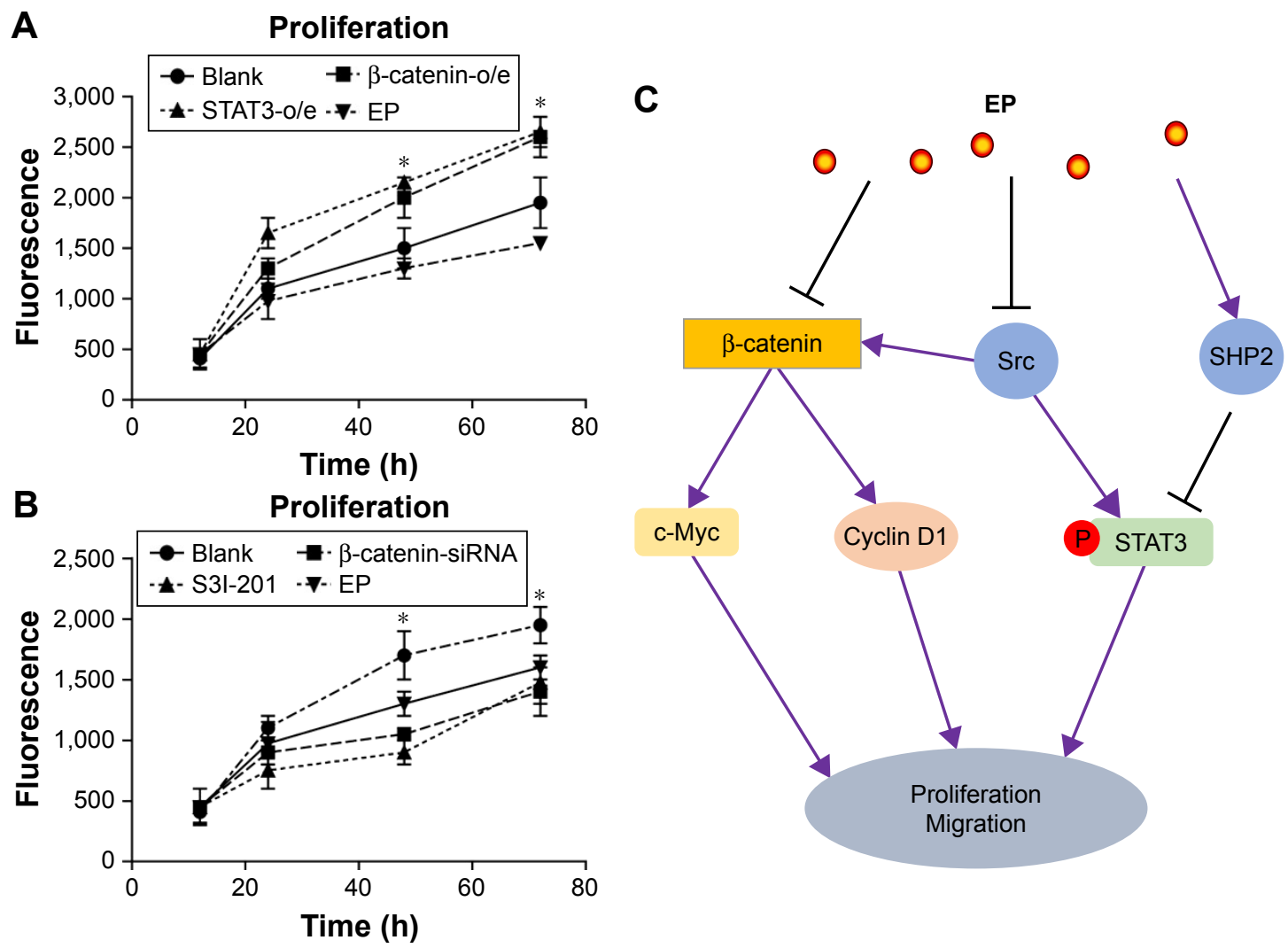

Figure 4 Multiple antitumor mechanisms of EP in ovarian cancer cells.

Notes: (A) Overexpression of either $\beta$-catenin or STAT3 up-regulated the proliferation of OVCAR-3 cells. (B) Knock-down of $\beta$-catenin or inhibition of STAT3 using S3I201 impaired cell viability, and EP treatment showed comparable cytotoxic effect with $\beta$-catenin-siRNA or STAT3-inhibition. $* P<0.05$. Therefore, our results suggested that both $\beta$-catenin and STAT3 were implicated in the antitumor mechanisms (C).

Abbreviations: EP, ergosterol peroxide; o/e, overexpression. 
to oncogenesis by modulating angiogenesis, ${ }^{30}$ and our results showed that EP can down-regulate the STAT3 activity. Taken together, it is highly possible that EP may also attenuate tumor angiogenesis, which needs more experimental evidence.

To date, all the studies about the antitumor effects of EP are based on cellular experiments. One of the reasons for lack of clinical perspective studies is the complicated components in medicinal mushrooms, and the isolation and purification of EP is not easy. However, Li et al recently reported the chemical synthesis of EP, which possesses a comparable antitumor effect to G. lucidum extracts. ${ }^{13}$ The successful synthesis makes it possible for further animal and clinical studies to verify the specific antitumor effects of EP. Our study provided initial evidence for the potential of EP in ovarian cancer treatment.

\section{Conclusion}

EP shows promising antitumor effects on ovarian cancer cells, which are mediated by both $\beta$-catenin and STAT3 pathways.

\section{Disclosure}

The authors report no conflicts of interest in this work.

\section{References}

1. Jemal A, Siegel R, Xu J, Ward E. Cancer statistics, 2010. CA Cancer J Clin. 2010;60(5):277-300.

2. Ferte C, Andre F, Soria JC. Molecular circuits of solid tumors: prognostic and predictive tools for bedside use. Nat Rev Clin Oncol. 2010; 7(7):367-380.

3. du Bois A, Luck HJ, Meier W, et al. A randomized clinical trial of cisplatin/paclitaxel versus carboplatin/paclitaxel as first-line treatment of ovarian cancer. J Natl Cancer Inst. 2003;95(17):1320-1329.

4. Colombo PE, Fabbro M, Theillet C, Bibeau F, Rouanet P, Ray-Coquard I. Sensitivity and resistance to treatment in the primary management of epithelial ovarian cancer. Crit Rev Oncol Hematol. 2014;89(2): 207-216.

5. Wang CW, Hsu WH, Tai CJ. Antimetastatic effects of cordycepin mediated by the inhibition of mitochondrial activity and estrogen-related receptor alpha in human ovarian carcinoma cells. Oncotarget. 2017; 8(2):3049-3058.

6. Huang TT, Wu SP, Chong KY, et al. The medicinal fungus Antrodia cinnamomea suppresses inflammation by inhibiting the NLRP3 inflammasome. J Ethnopharmacol. 2014;155(1):154-164.

7. Liu DL, Li YJ, Yang DH, et al. Ganoderma lucidum derived ganoderenic acid $\mathrm{B}$ reverses $\mathrm{ABCB} 1$-mediated multidrug resistance in HepG2/ADM cells. Int J Oncol. 2015;46(5):2029-2038.

8. Sliva D, Loganathan J, Jiang J, et al. Mushroom Ganoderma lucidum prevents colitis-associated carcinogenesis in mice. PLoS One. 2012; 7(10):e47873.

9. Wu QP, Xie YZ, Deng Z, et al. Ergosterol peroxide isolated from Ganoderma lucidum abolishes microRNA miR-378-mediated tumor cells on chemoresistance. PLoS One. 2012;7(8):e44579.

10. Zhao S, Ye G, Fu G, Cheng JX, Yang BB, Peng C. Ganoderma lucidum exerts anti-tumor effects on ovarian cancer cells and enhances their sensitivity to cisplatin. Int J Oncol. 2011;38(5):1319-1327.
11. Han J, Sohn EJ, Kim B, et al. Upregulation of death receptor 5 and activation of caspase $8 / 3$ play a critical role in ergosterol peroxide induced apoptosis in DU 145 prostate cancer cells. Cancer Cell Int. 2014;14(1):117.

12. Kobori M, Yoshida M, Ohnishi-Kameyama M, Shinmoto H. Ergosterol peroxide from an edible mushroom suppresses inflammatory responses in RAW264.7 macrophages and growth of HT29 colon adenocarcinoma cells. Br J Pharmacol. 2007;150(2):209-219.

13. Li X, Wu Q, Bu M, et al. Ergosterol peroxide activates Foxo3-mediated cell death signaling by inhibiting AKT and c-Myc in human hepatocellular carcinoma cells. Oncotarget. 2016;7(23):33948-33959.

14. Rhee YH, Jeong SJ, Lee HJ, et al. Inhibition of STAT3 signaling and induction of SHP1 mediate antiangiogenic and antitumor activities of ergosterol peroxide in U266 multiple myeloma cells. BMC Cancer. 2012;12:28.

15. Liu H, Zhang Q, Li K, et al. Prognostic significance of USP33 in advanced colorectal cancer patients: new insights into beta-arrestindependent ERK signaling. Oncotarget. 2016;7(49):81223-81240.

16. Guo S, Liu HD, Liu YF, Liu L, Sun Q, Cui XJ. Hepatoma-derived growth factor: a novel prognostic biomarker in intrahepatic cholangiocarcinoma. Tumour Biol. 2015;36(1):353-364.

17. Xu Y, Yang X, Li Z, et al. Sprouty2 correlates with favorable prognosis of gastric adenocarcinoma via suppressing FGFR2-induced ERK phosphorylation and cancer progression. Oncotarget. 2017;8(3): 4888-4900.

18. Clevers H, Nusse R. Wnt/beta-catenin signaling and disease. Cell. 2012; 149(6):1192-1205.

19. Yu H, Lee H, Herrmann A, Buettner R, Jove R. Revisiting STAT3 signalling in cancer: new and unexpected biological functions. Nat Rev Cancer. 2014;14(11):736-746.

20. Santoni M, Massari F, Del Re M, et al. Investigational therapies targeting signal transducer and activator of transcription 3 for the treatment of cancer. Expert Opin Investig Drugs. 2015;24(6):809-824.

21. Trusolino L, Bertotti A, Comoglio PM. MET signalling: principles and functions in development, organ regeneration and cancer. Nat Rev Mol Cell Biol. 2010;11(12):834-848.

22. Duarte N, Ramalhete C, Varga A, Molnar J, Ferreira MJ. Multidrug resistance modulation and apoptosis induction of cancer cells by terpenic compounds isolated from Euphorbia species. Anticancer Res. 2009; 29(11):4467-4472.

23. Li N, Hu YL, He CX, et al. Preparation, characterisation and anti-tumour activity of Ganoderma lucidum polysaccharide nanoparticles. J Pharm Pharmacol. 2010;62(1):139-144.

24. Wu SJ, Lu TM, Lai MN, Ng LT. Immunomodulatory activities of medicinal mushroom Grifola frondosa extract and its bioactive constituent. Am J Chin Med. 2013;41(1):131-144.

25. Dai J, Miller MA, Everetts NJ, et al. Elimination of quiescent slowcycling cells via reducing quiescence depth by natural compounds purified from Ganoderma lucidum. Oncotarget. 2017;8(8):13770-13781.

26. Russo A, Cardile V, Piovano M, Caggia S, Espinoza CL, Garbarino JA. Pro-apoptotic activity of ergosterol peroxide and (22E)-ergosta-7,22dien-5alpha-hydroxy-3,6-dione in human prostate cancer cells. Chem Biol Interact. 2010;184(3):352-358.

27. Kang JH, Jang JE, Mishra SK, et al. Ergosterol peroxide from Chaga mushroom (Inonotus obliquus) exhibits anti-cancer activity by downregulation of the beta-catenin pathway in colorectal cancer. $J$ Ethnopharmacol. 2015;173:303-312.

28. Cao R, Eriksson A, Kubo H, Alitalo K, Cao Y, Thyberg J. Comparative evaluation of FGF-2-, VEGF-A-, and VEGF-C-induced angiogenesis, lymphangiogenesis, vascular fenestrations, and permeability. Circ Res. 2004;94(5):664-670.

29. Valtola R, Salven P, Heikkilä P, et al. VEGFR-3 and its ligand VEGF-C are associated with angiogenesis in breast cancer. Am J Pathol. 1999; 154(5):1381-1390.

30. Gurbuz V, Konac E, Varol N, et al. Effects of AG490 and S3I-201 on regulation of the JAK/STAT3 signaling pathway in relation to angiogenesis in TRAIL-resistant prostate cancer cells in vitro. Oncol Lett. 2014;7(3):755-763. 


\section{Publish your work in this journal}

OncoTargets and Therapy is an international, peer-reviewed, open access journal focusing on the pathological basis of all cancers, potential targets for therapy and treatment protocols employed to improve the management of cancer patients. The journal also focuses on the impact of management programs and new therapeutic agents and protocols on

patient perspectives such as quality of life, adherence and satisfaction. The manuscript management system is completely online and includes a very quick and fair peer-review system, which is all easy to use. Visit http://www.dovepress.com/testimonials.php to read real quotes from published authors.

Submit your manuscript here: http://www.dovepress.com/oncotargets-and-therapy-journal 